\title{
THE MICROLOCAL SPECTRUM CONDITION AND WICK POLYNOMIALS OF FREE FIELDS ON CURVED SPACETIMES
}

\author{
R. BRUNETTI ${ }^{(1)}$, K. FREDENHAGEN ${ }^{(2)}$ AND M. KÖHLER ${ }^{(2)}$ \\ ${ }^{(1)}$ DIP. DI SCIENZE FISICHE \\ UNIVERSITÀ DI NAPOLI "FEDERICO II" \\ PAD. 19, MOSTRA D'OLTREMARE \\ I-80125 NAPOLI, ITALY \\ (2) II. INSTITUT FÜR THEORETISCHE PHYSIK, \\ UNIVERSITÄT HAMBURG \\ LURUPER CHAUSSEE 149 \\ D-22761 HAMBURG, GERMANY
}

PACS numbers: 1110, 0460, 0230

\begin{abstract}
Quantum fields propagating on a curved spacetime are investigated in terms of microlocal analysis. We discuss a condition on the wave front set for the corresponding n-point distributions, called "microlocal spectrum condition" $(\mu \mathrm{SC})$. On Minkowski space, this condition is satisfied as a consequence of the usual spectrum condition. Based on Radzikowski's determination of the wave front set of the two-point function of a free scalar field, satisfying the Hadamard condition in the Kay and Wald sense, we construct in the second part of this paper all Wick polynomials including the energy-momentum tensor for this field as operator valued distributions on the manifold and prove that they satisfy our microlocal spectrum condition.
\end{abstract}

Date: July 25, 2018.

e-mail: brunetti@axpna1.na.infn.it.

e-mail: i02fre@dsyibm.desy.de.

e-mail: mkoehler@x4u.desy.de. 


\section{INTRODUCTION}

Quantum Field Theory on curved spacetime (QFT on CST) describes quantum fields propagating under the influence of an external gravitational field. The main problem which has to be resolved in this setting is an appropriate formulation of stability. On Minkowski space, stability is expressed by the requirement of positive energy, i.e., the generators of time-like translations are represented as positive operators on some distinguished Hilbert space. In the absence of a time-like Killing vector field a corresponding condition can not be formulated and there exists no preferred Hilbert space. These difficulties can best be treated in the algebraic approach to quantum field theory HK64. In this approach, the formulation of a particular model can be divided into two steps. In the first step an algebra of observables is constructed in terms of commutation or anticommutation relations. This step was performed by Dimock [Dim80, Dim82, Dim92] for the free scalar, Dirac and electro-magnetic fields on globally hyperbolic spacetimes. In a second step, a class of states with suitable stability properties has to be found. States are here considered as expectation functionals, i.e., normalized positive linear functionals on the algebra of observables. Via the GNS construction each state induces a representation of the observable algebra in a Hilbert space with a cyclic vector but different states may lead to inequivalent representations.

One approach to characterize the class of states mentioned above is given by the "scaling limit criterion" and the "principle of local definiteness" introduced by Haag, Narnhofer and Stein [HNS84] and further investigated by Fredenhagen and Haag in [FH87]. Both characterizations are designed for states on general quantum field theoretical models on arbitrary spacetimes. On the other hand, for linear free models the so-called Hadamard states are believed to be physical states since the work of DeWitt and Brehme [DB60. See Kay and Wald KW91 for a precise definition. These states are characterized by the short distance behaviour of their two-point distributions [FSW78]: The singularity structure of the latter has to satisfy the Hadamard condition, in particular it is fixed by the underlying geometry. It was shown in Ver94 that these states satisfy the condition of local definiteness and that their local von Neumann algebras are factors of type III $_{1}$ (see also Wol92). Finally, in Hadamard states the expectation value of the stress energy tensor had successfully been renormalized Wal78]. Unfortunately the Hadamard condition is restricted to linear (free) fields.

Radzikowski showed in Rad92 (see also [Köh95]) that the global Hadamard condition can equivalently be formulated in terms of wave front sets. This formulation allows the powerful mathematical techniques of microlocal analysis developed in Hör71, DH72 to be used in QFT on CST. Indeed, one of us showed in Köh95 that the product of two scalar fields on a manifold is a well defined Wightman field on the GNS Hilbert space of any globally Hadamard product state. In this work we show a similar result for Wick-powers of scalar fields on a manifold. Moreover -using wave front sets- we propose a microlocal spectrum condition $(\mu \mathrm{SC})$ for all n-point distributions of a state, which is a generalization of the usual spectrum condition to manifolds. The first formulation of such a wave front set spectrum condition is due to Radzikowski Rad92]. However, his proposal needed to be modified, since free fields and products of free fields respectively provided counterexamples for his definition. It is also shown below that all Wick-powers of a free scalar field satisfy our $\mu \mathrm{SC}$. This gives, besides the product of two different scalar fields, another 
(nontrivial) example for a Wightman field satisfying our new condition. We expect the $\mu \mathrm{SC}$ to be an important ingredient for the formulation of quantum field theory on curved spacetimes, as well as a valuable tool for a rigorous formulation of perturbation theory on manifolds. In addition it might even deepen our understanding of the Wightman theory on Minkowski space and provide a local characterization of interaction.

The organization of this work is as follows. After this introduction some fundamental definitions and results of Hörmander's microlocal analysis are restated, i.e., the definition of wave front sets, theorems on the multiplication of distributions and composition of distribution valued operators respectively. These mathematical preliminaries are followed by a section on Hadamard states, in which Radzikowski's wave front characterization of these states is presented. In section t we propose our new $\mu \mathrm{SC}$ and show some fundamental consequences of our definition. Finally in the last section, Wick polynomials of a free scalar field are constructed. It is shown that they are (nontrivial) examples for new Wightman fields on a manifold.

\section{Mathematical Preliminaries}

This section is mainly written with the purpose to give an as much as possible self-contained introduction to some definitions and results of Hörmander's microlocal analysis, which will be needed in the sequel of this paper. Following the definition of the wave front set of a distribution, we recall Hörmander's results on the multiplication of distributions, which extends to the composition of distribution valued operators. For further details on this mathematical subject and the proofs of all Theorems stated below in this section, we refer the interested reader to the monograph of Hörmander Hör83 or to the original literature (i.e., Hör71, DH72]).

The theory of wave front sets was developed in the seventies by Hörmander together with Duistermaat Hör71, DH72], following a work of Sato [Sat69, Sat70]. The mathematicians use wave front sets (WF) mainly as a tool in partial differential

equations. Wave front sets are refinements of the notion of the singular support of a distribution. One of the main reasons for using them in favor of the latter is that they provide a simple characterization for the existence of products of distributions and eliminate the difference between local and global results. It is interesting that Duistermaat and Hörmander [DH72] have found a link between their microlocal analysis and quantum field theory, but their results apparently were seldom used in the physical literature.

The main purpose of microlocal analysis is to shift the study of singularities from the base space to the cotangent bundle. This is done by localizing the distribution around the singularity followed by analysing the result in "Fourier space".

Let $u \in \mathcal{D}^{\prime}\left(\mathbb{R}^{n}\right)$ be a distribution and let $\phi \in C_{0}^{\infty}(V)$ be a smooth function with support in $V \subset \mathbb{R}^{n}$. By a well known argument in distribution theory the Fourier transform of $\phi u$ yields a smooth function in frequency space for which the following relation holds:

$$
\widehat{\phi u}(\xi)=<u, e^{-i<\cdot, \xi>} \phi>,
$$

where $\langle\cdot, \cdot\rangle$ denotes dual pairing. This result implies

Lemma 2.1. Let $u \in \mathcal{D}^{\prime}(V)$ and let $W$ be an open subset of $V$. Then $\left.u\right|_{W} \in$ $C^{\infty}(W)$ if and only if for each $\phi \in C_{0}^{\infty}(W)$ and each integer $N \geq 0$ there is a 
constant $C_{\phi, N}$ such that

$$
\left|<u, e^{-i<\cdot, \xi>} \phi>\right| \leq C_{\phi, N}(1+|\xi|)^{-N} \quad \forall \xi \in \mathbb{R}^{n} .
$$

The singular support, $\operatorname{singsupp} u$, of $u \in \mathcal{D}^{\prime}(V)$ is defined to be the complement of the largest open subset of $V$ where $u$ is smooth. Motivated by the previous Lemma, a refinement of the notion of singular support which takes into account also the direction in which the Fourier transform does not strongly decay is the notion of wave front sets.

Definition 2.2. The wave front set, $\operatorname{WF}(u)$, of $u \in \mathcal{D}^{\prime}(V)$ is the complement in $V \times \mathbb{R}^{n} \backslash\{0\}$ of the set of points $\left(x_{0}, \xi_{0}\right)$ in $V \times \mathbb{R}^{n} \backslash\{0\}$ such that for some neighbourhood $U$ of $x_{0}$ and some conic neighbourhood $\Sigma$ of $\xi_{0}$ we have for each $\phi \in C_{0}^{\infty}(U)$ and each integer $N \geq 0$ a constant $C_{\phi, N}$ such that

$$
\left|<u, e^{-i<\cdot \xi\rangle} \phi>\right| \leq C_{\phi, N}(1+|\xi|)^{-N} \quad \forall \xi \in \Sigma .
$$

Note that a conic set $\Sigma$ is such that if $(x, \xi) \in \Sigma$ then $(x, t \xi) \in \Sigma$ for all $t>0$. The following Remarks can be easily proved using Lemma 2.1

\section{Remark .}

1. For $v \in \mathcal{D}^{\prime}(V), V$ an open subset of $\mathbb{R}^{n}$, with wave front set $\operatorname{WF}(v)$, the projection of the wave front set to the base point gives the singular support of $v$.

2. $\mathrm{WF}(v)$ is a closed subset of $V \times \mathbb{R}^{n} \backslash\{0\}$ since each point $(x, k) \notin \mathrm{WF}(v)$ has by definition an open neighbourhood in $V \times \mathbb{R}^{n} \backslash\{0\}$ consisting of such points, too.

3. For all smooth test functions $\phi$ with compact support $\mathrm{WF}(\phi v) \subset \mathrm{WF}(v)$.

4. For any distribution $v$ with wave front set $\mathrm{WF}(v)$, the wave front sets of its partial derivatives are contained in $\mathrm{WF}(v)$.

\section{Example 2.3.}

1. Let $f \in C^{\infty}(V) \subset \mathcal{D}^{\prime}(V)$ be a smooth function, then $\operatorname{WF}(f)=\emptyset$.

2. Consider the Dirac $\delta$-distribution on $\mathbb{R}^{2}$. Its easy to see that its wave front set is the following $\operatorname{WF}(\delta(x, y))=\left\{\left(x, k ; y, k^{\prime}\right) \in \mathbb{R}^{2} \times \mathbb{R}^{2 n} \backslash\{0\} \mid \quad x=y ; k=\right.$ $\left.-k^{\prime}\right\}$.

It is worth recalling that the set of normal coordinates $\left(x_{1}, \ldots, x_{n}, \xi_{1}, \ldots, \xi_{n}\right)$ of the cotangent bundle $T^{*} V$ over the base coordinates $\left(x_{1}, \ldots, x_{n}\right)$ in $V$ allows us to identify $V \times \mathbb{R}^{n}$ with $T^{*} V$ and to consider $\mathrm{WF}(u)$ as a subset of the cotangent bundle. Since the definition of wave front sets is local it can be lifted to manifolds. We give below an intrinsic definition of wave front sets.

Throughout this work a smooth manifold $M$ is a locally euclidean, Hausdorff, second countable topological space equipped with a smooth structure. Let $\Omega_{\alpha}(M)$ denotes the complex line bundle of densities of order $\alpha$ over a smooth manifold $M$, i.e., $\Omega_{\alpha}(M)=\cup_{x \in M} \Omega_{\alpha}\left(T_{x} M\right)$ where $T_{x} M$ denote the tangent space at $x$ of $M$ (see Dui73). Let then $C_{0}^{\infty}\left(M, \Omega_{1-\alpha}\right)$ be the vector space of compactly supported smooth sections of the line bundle $\Omega_{1-\alpha} \equiv \Omega_{1-\alpha}(M)$ equipped with the usual topology, then, $\mathcal{D}^{\prime}\left(M, \Omega_{\alpha}\right)$, the space of distribution densities of order $\alpha$ over a smooth manifold $M$, is defined as the space of all continuous linear forms on the space $C_{0}^{\infty}\left(M, \Omega_{1-\alpha}\right)$. For simplicity we call distribution densities of order zero distributions and denote their corresponding space by $\mathcal{D}^{\prime}(M)$. It is worth noting that the set of all smooth functions on $M$ is dense in $\mathcal{D}^{\prime}(M)$. 
Definition 2.4. Let $M$ be an n-dimensional smooth manifold with cotangent bundle $T^{*} M$ and take $u \in \mathcal{D}^{\prime}\left(M, \Omega_{\alpha}\right)$. The point $\left(x_{0}, k_{0}\right) \in T^{*} M \backslash\{0\}$ is called a regular directed point if and only if for all $s \geq 1$, for all $\lambda_{0} \in \mathbb{R}^{s}$ and for any function $\phi \in C^{\infty}\left(M \times \mathbb{R}^{s}, \mathbb{R}\right)$ such that $d_{x} \phi\left(x_{0}, \lambda_{0}\right)=k_{0}$ there exists a neighbourhood $V$ of $x_{0}$ in $M$ and a neighbourhood $\Lambda$ of $\lambda_{0}$ in $\mathbb{R}^{s}$ such that, for all $\rho \in C_{0}^{\infty}\left(V, \Omega_{1-\alpha}\right)$ and all $N \geq 0$, we have, uniformly in $\lambda \in \Lambda$,

$$
\left|<u, \rho e^{-\imath \tau \phi(\cdot, \lambda)}>\right|=0\left(\tau^{-N}\right) \quad \text { if } \quad \tau \longrightarrow \infty .
$$

The wave front set, $\operatorname{WF}(u)$, of $u \in \mathcal{D}^{\prime}\left(M, \Omega_{\alpha}\right)$ is now the complement in $T^{*} M \backslash$ $\{0\}$ of the set of all regular directed points of $u$. By localization and choice of a strictly positive density on $M$, such that one can identify test-densities with compact support with test-functions with compact support, the definition coincides with that given previously. Moreover, the same properties as in the Remark on page 1 hold on manifolds.

A useful application of wave front sets is the definition of products of distributions. Wave front sets provide a simple characterization for the existence of such products, which turns out to be sequentially continuous provided the wave front sets of the corresponding distributions are contained in a suitable cone in $T^{*} M \backslash\{0\}$.

Definition 2.5. Let $\Gamma$ be a closed cone in $T^{*} M \backslash\{0\}$ and let $\mathcal{D}_{\Gamma}^{\prime}\left(M, \Omega_{\alpha}\right)$ denote the subspace of distributional densities of order $\alpha$ with wave front set contained in $\Gamma$. A sequence $\left\{u_{j}\right\}$ of distributional densities in $\mathcal{D}_{\Gamma}^{\prime}\left(M, \Omega_{\alpha}\right)$ converges to a distribution $u \in \mathcal{D}_{\Gamma}^{\prime}\left(M, \Omega_{\alpha}\right)$ iff the following conditions hold;

1. $\left\{u_{j}\right\}$ converges to $u$ in $\mathcal{D}^{\prime}\left(M, \Omega_{\alpha}\right)$ (weakly),

2. for all $\left(x_{0}, k_{0}\right) \in\left(T^{*} M \backslash\{0\}\right) \backslash \Gamma$, there exists a density of order $(1-\alpha) \rho \in$ $C_{0}^{\infty}\left(M, \Omega_{1-\alpha}\right)$ with $\rho\left(x_{0}\right) \neq 0$, a conic neighbourhood $W$ of $k_{0}$ in $T^{*} M \backslash\{0\}$ and a function $\phi$ as in Definition 2.4 such that for all $N$,

$$
\sup _{\tau \in \mathbb{R}_{+}} \sup _{k \in W}\left[\tau^{N} \mid<u-u_{j}, \rho e^{-i \tau \phi(\cdot, k)}>1\right] \longrightarrow 0 \quad \text { if } j \longrightarrow \infty .
$$

It is worth noting that every subspace $\mathcal{D}_{\Gamma}^{\prime}\left(M, \Omega_{\alpha}\right)$ contains all smooth densities of order $\alpha$ with compact support. Moreover, let $u \in \mathcal{D}_{\Gamma}^{\prime}(M)$, then there exists a sequence $\left\{u_{j}\right\}$ of compactly supported smooth functions such that $u_{j} \rightarrow u$ in $\mathcal{D}_{\Gamma}^{\prime}(M)$. It is therefore possible to choose their supports in an arbitrary neighbourhood of the support of $u$.

Theorem 2.6. Let $M$ be a smooth manifold and let $\Gamma, \Sigma \in T^{*} M \backslash\{0\}$ be two closed cones, such that $\Gamma \oplus \Sigma:=\{(x, k+l) \mid(x, k) \in \Gamma ;(x, l) \in \Sigma\} \subseteq T^{*} M \backslash\{0\}$. Then the multiplication operator

$$
C_{0}^{\infty}\left(M, \Omega_{\alpha}\right) \times C_{0}^{\infty}\left(M, \Omega_{\beta}\right) \ni(u, v) \longmapsto u \cdot v \in C_{0}^{\infty}\left(M, \Omega_{\alpha+\beta}\right)
$$

extends to a unique sequentially continuous operator from $\mathcal{D}_{\Gamma}^{\prime}\left(M, \Omega_{\alpha}\right) \times \mathcal{D}_{\Sigma}^{\prime}\left(M, \Omega_{\beta}\right)$ to $\mathcal{D}_{\Lambda}^{\prime}\left(M, \Omega_{\alpha+\beta}\right)$, where $\Lambda:=(\Gamma \oplus \Sigma) \cup \Gamma \cup \Sigma$. In particular, one finds for the wave front sets:

$$
\mathrm{WF}(u \cdot v) \subseteq \mathrm{WF}(u) \oplus \mathrm{WF}(v) \cup \mathrm{WF}(u) \cup \mathrm{WF}(v) .
$$

Proof. In Hörmander Hör71, Theorem 2.5.10] this Theorem is proved for the case $\alpha=\beta=0$ only, but the result extends to arbitrary values of $\alpha$ and $\beta$ : Note first that every distribution density of order $\alpha$ can be written as a distribution times a smooth positive density of order $\alpha$. Using Hörmander's result the product of 
these distributions multiplied with the tensor product of the corresponding smooth densities yields a distributional density of order $\alpha+\beta$, which does not depend on the splitting done before. For the last statement recall that two smooth densities differ by a smooth function and that the multiplication operator is associative.

Remark . Note that for the existence of the product of two distributional densities $u$ and $v$ it is sufficient to check that $\mathrm{WF}(u) \oplus \mathrm{WF}(v)$ does not contain terms of the form $(x, 0)$.

2.1. The composition of distribution valued operators. In this subsection some properties for the composition of two distribution valued operators are recalled. Let $M$ and $\tilde{M}$ be two smooth manifolds. Consider an operator $K_{1}$ from $C_{0}^{\infty}\left(M, \Omega_{1}\right)$ to $\mathcal{D}^{\prime}\left(\tilde{M}, \tilde{\Omega}_{1}\right)$. By Schwartz nuclear Theorem this operator is in a one-to-one correspondence to a distribution density $\mathcal{K}_{1} \in \mathcal{D}^{\prime}\left(M \times \tilde{M}, \tilde{\Omega}_{1}\right)$, such that,

$$
<K_{1} \rho, \tilde{g}>=\mathcal{K}_{\infty}(\rho \otimes \tilde{g}) \quad \forall \rho \in C_{0}^{\infty}\left(M, \Omega_{1}\right), \tilde{g} \in C_{0}^{\infty}(\tilde{M}) .
$$

For the wave front sets of $K_{1}$ we introduce the following notations,

$$
\begin{aligned}
\mathrm{WF}\left(K_{1}\right) & :=\operatorname{WF}\left(\mathcal{K}_{1}\right) \\
\mathrm{WF}^{\prime}\left(K_{1}\right) & :=\left\{(x, h ; \tilde{x}, \tilde{h}) \mid(x, h ; \tilde{x},-\tilde{h}) \in \mathrm{WF}\left(K_{1}\right)\right\} \\
\mathrm{WF}_{\tilde{M}}\left(K_{1}\right) & :=\left\{(\tilde{x}, \tilde{h}) \mid(x, 0 ; \tilde{x}, \tilde{h}) \in \mathrm{WF}\left(K_{1}\right)\right\} .
\end{aligned}
$$

Consider now a second operator $K_{2}$ from $C_{0}^{\infty}\left(\tilde{M}, \tilde{\Omega}_{1}\right)$ to $\mathcal{D}^{\prime}(\tilde{\tilde{M}})$, where $\tilde{\tilde{M}}$ is a third smooth manifold, whose corresponding distribution is denoted by $\mathcal{K}_{2} \in$ $\mathcal{D}^{\prime}(\tilde{\tilde{M}} \times \tilde{M})$.

Theorem 2.7 ( Hör83, Theorem 8.2.14]). The composition $K_{2} \circ K_{1}$ is a well defined operator from $C_{0}^{\infty}\left(M, \Omega_{1}\right)$ to $\mathcal{D}^{\prime}(\tilde{M})$, provided that,

i) $K_{1}$ has proper support, i.e., the inverse image of any compact set under the projection $\operatorname{supp} K_{1} \ni(\tilde{y}, x) \mapsto x$ is compact,

ii) $\mathrm{WF}_{\tilde{M}}^{\prime}\left(K_{2}\right) \cap \mathrm{WF}_{\tilde{M}}\left(K_{1}\right)=\emptyset$.

For the wave front set of $K_{2} \circ K_{1}$ one finds:

$$
\begin{aligned}
\mathrm{WF}\left(K_{2} \circ K_{1}\right) \subseteq & \mathrm{WF}^{\prime}\left(K_{2}\right) \circ \mathrm{WF}\left(K_{1}\right) \\
& \cup\left(\mathrm{WF}_{\tilde{\tilde{M}}}\left(K_{2}\right) \times M \times\{0\}\right) \cup\left(\tilde{\tilde{M}} \times\{0\} \times \mathrm{WF}_{M}\left(K_{1}\right)\right),
\end{aligned}
$$

where

$$
\begin{array}{r}
\mathrm{WF}^{\prime}\left(K_{2}\right) \circ \mathrm{WF}\left(K_{1}\right)=\left\{(\tilde{\tilde{x}}, \tilde{\tilde{k}} ; x, k) \in\left(T^{*} \tilde{\tilde{M}} \times T^{*} M\right) \backslash\{0\} \mid(\tilde{\tilde{x}}, \tilde{\tilde{k}} ; \tilde{x}, \tilde{k}) \in \mathrm{WF}^{\prime}\left(K_{2}\right)\right. \\
\left.\quad \text { and }(\tilde{x}, \tilde{k} ; x, k) \in \mathrm{WF}\left(K_{1}\right) \quad \text { for some }(\tilde{x}, \tilde{k}) \in T^{*} \tilde{M}\right\} .
\end{array}
$$

Moreover, the composition is sequentially continuous, i.e., if $K_{2 \epsilon}$ is a sequence of smooth $\left(\mathrm{WF}\left(K_{2 \epsilon}\right)=\emptyset\right)$ operators with proper supports converging to $K_{2}$, then the composition $K_{2 \epsilon} \circ K_{1}$ converges to $K_{2} \circ K_{1}$, provided the latter composition exists.

To shorten the notation we will use the same symbol for the operator and the distribution density in what follows. The next Corollary will be useful in section 4 .

\footnotetext{
${ }^{1}$ The topology on the operators is the one inherited by that of the corresponding distribution densities.
} 
Corollary 2.8 ([Hör83, Theorem 8.2.13]). Let $K \in \mathcal{D}^{\prime}(M \times \tilde{M})$ denote an operator from $C_{0}^{\infty}\left(\tilde{M}, \tilde{\Omega}_{1}\right)$ to $\mathcal{D}^{\prime}(M)$. Then for $\rho \in C_{0}^{\infty}\left(\tilde{M}, \tilde{\Omega}_{1}\right) \operatorname{WF}(K(\cdot, \rho)) \subseteq$ $\mathrm{WF}_{\tilde{M}}(K)$.

\section{Hadamard states}

We describe quantum fields propagating on a four dimensional Lorentz manifold $\left(M, g_{a b}\right)$ in terms of the General Theory of Quantized Fields (See Fre92, Haa92, Wa194). The manifold is assumed to be globally hyperbolic, i.e., it admits spacelike Cauchy hypersurfaces. We deal with the Gårding-Wightman WG64 approach to quantum fields and its algebraic formulation by Borchers and Uhlmann (Bor62, Uhl62 ). We refer the reader to the cited literature for details, but nonetheless let us give some ideas for completeness. The Borchers-Uhlmann algebra $\mathcal{B}$ for the scalar field mentioned above is the tensor algebra over $C_{0}^{\infty}\left(M, \Omega_{1}\right)$. A state $\omega$, defined as a positive linear functional over $\mathcal{B}$ consists of a hierarchy of m-point distributions $\omega=\left\{\omega_{m}\right\}_{m \in \mathbb{N}}$. Every state satisfying local commutativity fixes -via the following GNS-construction Theorem- a Hilbert space, a "vacuum vector" and a representation of the algebra $\mathcal{B}$ thus links the algebraic approach to the Hilbert space setting of Gårding and Wightman.

Theorem 3.1 (GNS-reconstruction). For every state $\omega=\left\{\omega_{m}\right\}_{m \in \mathbb{N}}$ on the scalar Borchers-Uhlmann algebra there is a GNS-tupel $\left(\mathcal{H}_{\omega}, \mathcal{D}_{\omega}, \phi_{\omega}, \Omega_{\omega}\right)$, unique up to unitary equivalence, such that for each $m \geq 1$ and any test densities $f_{1}, \ldots, f_{m} \in$ $C_{0}^{\infty}\left(M, \Omega_{1}\right)$

$$
\omega_{m}\left(f_{1}, \ldots, f_{m}\right)=\left(\Omega_{\omega}, \phi_{\omega}\left(f_{1}\right) \cdots \phi_{\omega}\left(f_{m}\right) \Omega_{\omega}\right) .
$$

Recall that a GNS-tupel $\left(\mathcal{H}_{\omega}, \mathcal{D}_{\omega}, \phi_{\omega}, \Omega_{\omega}\right)$ satisfies the following properties:

1. $\mathcal{H}_{\omega}$ is a separable Hilbert space, $\mathcal{D}_{\omega}$ is a dense subspace of $\mathcal{H}_{\omega}$ and the GNSvacuum $\Omega_{\omega}$ is a distinguished vector in $\mathcal{H}_{\omega}$.

2. The fields $\phi_{\omega}$ are operator valued distributions, i.e., for all $\Phi, \Psi \in \mathcal{D}_{\omega}$ the linear mapping

$$
\left(\Psi, \phi_{\omega}(\cdot) \Phi\right): C_{0}^{\infty}\left(M, \Omega_{1}\right) \ni f \mapsto\left(\Psi, \phi_{\omega}(f) \Phi\right)
$$

is in $\mathcal{D}^{\prime}(M)$.

3. The subspace $\mathcal{D}_{\omega}$ contains $\Omega_{\omega}$ and is an invariant domain for the fields, i.e., for each $f \in C_{0}^{\infty}\left(M, \Omega_{1}\right)$ the domain of $\phi_{\omega}(f)$ contains $\mathcal{D}_{\omega}$ and $\phi_{\omega}(f) \mathcal{D}_{\omega} \subset \mathcal{D}_{\omega}$.

4. The fields are hermitian, i.e., for each $f \in C_{0}^{\infty}\left(M, \Omega_{1}\right)$, the domain of the adjoint of $\phi_{\omega}(f)$-denoted by $\phi_{\omega}^{*}(f)$ - contains $\mathcal{D}_{\omega}$ and $\phi_{\omega}^{*}(f) \supset \phi_{\omega}(\bar{f})$, where the bar denotes complex conjugation.

5. The subspace $\mathcal{D}_{\omega}$ is generated by applying finitely many smeared field operators to the GNS-vacuum.

If $\phi_{\omega}$ satisfies the Klein-Gordon equation and its commutator is given by

$$
\left[\phi_{\omega}(f), \phi_{\omega}(g)\right]=E(f \otimes g) \quad \forall f, g \in C_{0}^{\infty}\left(M, \Omega_{1}\right)
$$

where $E$ is the difference between the advanced and retarded fundamental solution of the Klein-Gordon operator, we call $\omega$ a state of the Klein-Gordon field over M. It was already mentioned in the introduction, that not all states $\omega$ are believed to be physically meaningful. A condition which physically admissible states should satisfy is the Hadamard condition ([DB60]) which was intensively studied by various authors (see the references in Fulling's book Ful89]). A mathematically precise 
definition of the Hadamard condition in terms of boundary values of certain complex valued functions was given recently by Kay and Wald KW91. Radzikowski discovered in his thesis that, equivalently, Hadamard states can be characterized in terms of their wave front sets. Using his results Junker Jun95] has been able to construct Hadamard states for free scalar fields on arbitrary globally hyperbolic spacetimes. In this section we recall Radzikowski's wave front set characterization.

Definition 3.2. Let $\omega$ be a quasifree state of the Klein-Gordon field over a globally hyperbolic manifold $\left(M, g_{a b}\right)$. Then $\omega$ is a Hadamard state if and only if its twopoint distribution $\omega_{2}$ has wave front set

$$
\mathrm{WF}\left(\omega_{2}\right)=\left\{\left(x_{1}, k_{1}\right),\left(x_{2},-k_{2}\right) \in T^{*} M^{2} \backslash\{0\} \mid\left(x_{1}, k_{1}\right) \sim\left(x_{2}, k_{2}\right) ; k_{1}^{0} \geq 0\right\}
$$

where $\left(x_{1}, k_{1}\right) \sim\left(x_{2}, k_{2}\right)$ means that there exists a light-like geodesic $\gamma$ connecting $x_{1}$ and $x_{2}$ with cotangent vectors $k_{1}$ at $x_{1}$ and $k_{2}$ at $x_{2}$.

Recall that a state $\omega$ is called quasifree iff all its odd m-point distributions vanish and

$$
\omega_{m}\left(x_{1}, \ldots, x_{m}\right)=\sum_{P} \prod_{r} \omega_{2}\left(x_{(r, 1)}, x_{(r, 2)}\right) .
$$

Here $P$ denotes a partition of the set $\{1, \cdots, m\}$ into subsets which are pairings of points, labeled by $r$. Note that the ordering of the points in $\omega_{2}$ is preserved, e.g. $(r, 1)<(r, 2)$ and no two arguments are identical. The latter fact ensures the existence of the product $\prod_{r}$ whenever $\omega_{2}\left(x_{i}, x_{j}\right)$ are distributions.

For the wave front set of $\omega_{m}$ one finds using Theorem 2.6 and Eqn. (价)

$$
\mathrm{WF}\left(\omega_{m}\right) \subseteq\left(\bigcup_{Q} \bigoplus_{r \in Q} \mathrm{WF}\left(\omega_{2}^{r}\right)\right),
$$

where $Q$ denotes a nonempty set of disjoint pairs and where $\omega_{2}^{r}$ is the two-point distribution in the varables $x_{(r, 1)}, x_{(r, 2)}$ considered as a distribution on $M^{n}$ and hence has wave front set

$$
\begin{aligned}
\mathrm{WF}\left(\omega_{2}^{r}\right)= & \left\{\left(x_{1}, 0 ; \ldots ; x_{(r, 1)}, k_{(r, 1)} ; \ldots ; x_{(r, 2)}, k_{(r, 2)} ; \ldots ; x_{n}, 0\right) \mid\right. \\
& \left.\left(x_{(r, 1)}, k_{(r, 1)} ; x_{(r, 2)}, k_{(r, 2)}\right) \in \mathrm{WF}\left(\omega_{2}\right)\right\} .
\end{aligned}
$$

Using this characterization one can prove the following

Corollary 3.3 (Köh95). Let $\omega^{1}$ and $\omega^{2}$ be quasifree Hadamard states for two massive Klein-Gordon fields propagating on a globally hyperbolic spacetime $\left(M, g_{a b}\right)$. Then the pointwise product of the corresponding m-point distributions exists and gives rise to a new Wightman field on this spacetime.

\section{Microlocal SPECtrum CONDition}

In this section we propose a condition on the wave front sets of states of a quantum field on a smooth manifold $M$, which generalizes the usual Minkowski space spectrum condition to curved spacetimes. The idea to use wave front sets for a formulation of some kind of spectrum condition is due to Radzikowski Rad92. For a motivation recall that the Hadamard condition can be formulated as a condition on the wave front set of the corresponding two-point distribution. Since Eqn. (3) restricts the singular support of $\omega_{2}\left(x_{1}, x_{2}\right)$ to points $x_{1}$ and $x_{2}$ which are null 
related, $\omega_{2}$ is smooth for all other points. The smoothness for space-like related points is known to be true for quantum field theories on Minkowski space satisfying the true spectrum condition by the Bargmann-Hall-Wightman Theorem. For timelike related points however a similar general prediction on the smoothness does not exist. In order to include possible singularities at time-like related points, Radzikowski extended in Rad92 the right-hand side of Eqn. (3) to all causally related points; he proposed that the wave front set of the two-point distributions of any physical reasonable state should be contained in this extended set. He called this proposal the "wave front set spectrum condition" (WFSSC). He also proposed a WFSSC for higher m-point distributions and linked both to the scaling limit condition of [HNS84, FH87]: Both definitions imply the true spectrum condition in the scaling limit if this limit exists (Theorem 4.11 of [Rad92]). Unfortunately it can be shown that the m-point distributions for $m>2$ associated to a quasifree Hadamard state of a scalar field on a globally hyperbolic spacetime do not satisfy his WFSSC in general. Moreover it was shown in Köh95 that the distributional product of two different fields gives rise to counterexamples even for his two point WFSSC. This result is also true for the Wick powers constructed below. Thus, his original WFSSC needs to be modified.

Below we propose a wave front set spectrum condition, which is satisfied by the examples mentioned above. For a more compact notation some definitions from graph theory are used: Let $\mathcal{G}_{n}$ denote the set of all finite graphs with vertices $\{1, \ldots, n\}$, such that for every element $G \in \mathcal{G}_{n}$ all edges occur in both admissible directions. An immersion of a graph $G \in \mathcal{G}_{n}$ into some Lorentz manifold $M$ is an assignment of the vertices of $G$ to points in $M, \nu \rightarrow x(\nu)$, and of the edges of $G$ to piecewise smooth 2 curves in $M, e \rightarrow \gamma(e)$ with source $s(\gamma(e))=x(s(e))$ and range $r(\gamma(e))=x(r(e))$ respectively, together with a covariantly constant causal covector field $k_{e}$ on $\gamma\left(\nabla k_{e}=0\right)$, such that

1. If $e$ is an edge from $\nu$ to $\nu^{\prime}$ then $\gamma(e)$ connects $x(\nu)$ and $x\left(\nu^{\prime}\right)$,

2 . If $e^{-1}$ denotes the edge with opposite direction as $e$, then the corresponding curve $\gamma\left(e^{-1}\right)$ is the inverse of $\gamma(e)$,

3. For every edge $e$ from $\nu$ to $\nu^{\prime}, k_{e}$ is directed towards the future whenever $\nu<\nu^{\prime}$,

4. $k_{e^{-1}}=-k_{e}$.

(Compare Figure 田)

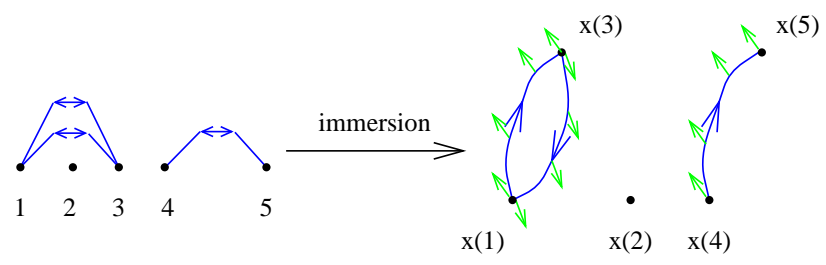

Figure 1. An immersion of a graph

We propose the following criterium as a substitute of the usual spectrum condition for field theories over a globally hyperbolic manifold,

\footnotetext{
${ }^{2}$ Replacing "smooth" by "causal" or "light-like" yields stronger versions of the Microlocal Spectrum Condition (Definition 4.1 below).
} 
Definition 4.1 ( $\mu \mathrm{SC})$. A state $\omega$ with m-point distributions $\omega_{m}$ is said to satisfy the Microlocal Spectrum Condition ( $\mu \mathrm{SC}$ ) if and only if, for any $m$,

$$
\begin{aligned}
& \operatorname{WF}\left(\omega_{m}\right) \\
& \subseteq\left\{\left(x_{1}, k_{1} ; \ldots ; x_{m}, k_{m}\right) \in T^{*} M^{m} \backslash\{0\} \mid \quad \exists G \in \mathcal{G}_{m}\right.
\end{aligned}
$$

and an immersion $(x, \gamma, k)$ of $G$, such that

$$
\begin{aligned}
\text { (i) } x_{i} & =x(i) \quad \forall i=1, \ldots, m \\
\text { (ii) } k_{i} & \left.=\sum_{\substack{e \\
s(e)=i}} k_{e}\left(x_{i}\right)\right\}=\Gamma_{m} .
\end{aligned}
$$

Remark. For every set of base points $\left(x_{1}, \ldots, x_{m}\right) \in \operatorname{singsupp}\left(\omega_{m}\right)$ the first non zero direction $k_{l}$ in the wave front set is future directed.

Lemma 4.2. The sets $\Gamma_{m}$ are stable under addition for all $m \in \mathbb{N}$, i.e.,

$$
\Gamma_{m} \oplus \Gamma_{m} \subseteq \Gamma_{m}
$$

Proof. Let $(\boldsymbol{x}, \boldsymbol{k})=\left(x_{1}, k_{1} ; \ldots, x_{m}, k_{m}\right)$ and $(\boldsymbol{x}, \tilde{\boldsymbol{k}})=\left(x_{1}, \tilde{k}_{1} ; \ldots, x_{m}, \tilde{k}_{m}\right)$ be two points in $\Gamma_{m}$ with corresponding graphs and immersion $G,(x, \gamma, k)$ and $\tilde{G},(x, \tilde{\gamma}, \tilde{k})$ respectively. To prove the assertion, it must be shown, that the point $\left(\boldsymbol{x}, \boldsymbol{k}_{+}\right)=$ $\left(x_{1}, k_{1}+\tilde{k}_{1} ; \ldots, x_{m}, k_{m}+\tilde{k}_{m}\right)$ is contained in $\Gamma_{m}$. Let $G_{+} \in \mathcal{G}_{m}$ denote a graph with $m$ vertices, whose set of edges is the disjoint union of the sets of edges of $G$ and $\tilde{G}$ respectively. The immersions of the latter two graphs yield an immersion $\left(\boldsymbol{x}, \boldsymbol{k}_{+}\right)$for $G_{+}$, which satisfies i) and ii) of Definition 4.1. Hence it remains to be shown, that $\left(\boldsymbol{x}, \boldsymbol{k}_{+}\right)$is contained in $T^{*}\left(M^{m}\right) \backslash\{0\}$, i.e., that $k_{i}+\tilde{k}_{i} \neq 0$ for some $i=1, \ldots, m$. By definition of $\Gamma_{m}$, both $\boldsymbol{k}$ and $\tilde{\boldsymbol{k}}$ are nonzero and the first nonvanishing entries $k_{i}$ of $\boldsymbol{k}$ and $\tilde{k}_{j}$ of $\tilde{\boldsymbol{k}}$ are elements of $\bar{V}_{+} \backslash\{0\}$. Hence, for $l=\min (i, j)$ we find $k_{l}+\tilde{k}_{l} \in \bar{V}_{+} \backslash\{0\}$.

To show that there exist non trivial states which satisfy our Definition we prove the following

Proposition 4.3. Let $\omega$ denote a quasifree Hadamard state for the Klein-Gordon field on a globally hyperbolic manifold $\left(M, g_{a b}\right)$, then $\omega$ satisfies the $\mu S C$.

Proof. Note first that all odd m-point distributions vanish by assumption, hence $\omega_{m}$ satisfies the $\mu \mathrm{SC}$ trivially for odd $\mathrm{m}$. Consider now the two-point distribution $\omega_{2}$. Its wave front set is given explicitly by Eqn. (3) and obviously satisfies the $\mu \mathrm{SC}$. For a general m-point distribution consider the representation given by Eqn. (4), which states that $\omega_{m}$ is a sum of tensor products of two-point distributions. Hence, there exists a disconnected graph $G_{m} \in \mathcal{G}_{m}$ together with an immersion $(x, \gamma, k)$, such that (i) and (ii) of Definition 4.1 are satisfied, i.e., $G_{m}$ consists of subgraphs $G_{2} \in \mathcal{G}_{2}$, such that the immersion $(x, \gamma, k)$ restricted to these subgraphs is compatible with the wave front set of the corresponding two-point distribution.

Remark . 
- A complete analogue of this Proposition should be valid in the case of the Dirac equation, since Hadamard states for the latter are obtainable by applying the adjoint of the Dirac operator to a suitable (auxiliary) Hadamard state of the 'squared' Dirac equation. For fixed spinor indices the wave front set of the latter is contained in the r.h.s. of Eqn. (3) and derivatives do not enlarge the wave front set.

- Junker Jun95 rigorously proved recently that the adiabatic vacuum states on a Robertson-Walker spacetime are globally Hadamard states. Hence they satisfy the $\mu \mathrm{SC}$, too.

States satisfying the $\mu \mathrm{SC}$ obey some nice properties outlined in the following Theorems.

Theorem 4.4. Let $\omega^{1}$ and $\omega^{2}$ be two states satisfying the $\mu S C$. Then the pointwise products of their corresponding n-point distributions exist and define a new state satisfying the $\mu S C$.

Proof. To prove the existence of the product, it is -by Theorem 2.6- sufficient to show that the sums of $\mathrm{WF}\left(\omega_{m}^{i}\right)(i=1,2)$ do not intersect with the zero section. Now by assumption $\operatorname{WF}\left(\omega_{m}^{1}\right)$ and $\operatorname{WF}\left(\omega_{m}^{2}\right)$ are both contained in the set $\Gamma_{m}$, which -by Lemma 4.2 is stable under addition. Hence $\mathrm{WF}\left(\omega_{m}^{1}\right) \oplus \mathrm{WF}\left(\omega_{m}^{2}\right) \subseteq \Gamma_{m} \subset$ $T^{*} M^{m} \backslash\{0\}$, which ensures the existence of the products and also implies that they satisfy the $\mu \mathrm{SC}$.

In order to prove that these new m-point distributions yield a state, i.e., satisfy Wightman positivity, we consider the tensor product of $\omega^{1}$ and $\omega^{2}$. This is a state on the Borchers-Uhlmann algebra of two commuting scalar fields. Positivity for this state implies for every test densities $f_{j} \in C_{0}^{\infty}\left(M^{j}, \Omega_{1}^{j}\right), g \in C_{0}^{\infty}\left(M^{2}, \Omega_{1}^{2}\right)$,

$$
\begin{aligned}
0 \leq \sum_{m, n} \int & \left(\omega_{1}\right)_{n+m}\left(x_{n}, \ldots, x_{1}, x_{1}^{\prime}, \ldots, x_{m}^{\prime}\right)\left(\omega_{2}\right)_{n+m}\left(y_{n}, \ldots, y_{1}, y_{1}^{\prime}, \ldots, y_{m}^{\prime}\right) \\
& \times \overline{f_{n}\left(x_{1}, \ldots, x_{n}\right)} f_{m}\left(x_{1}^{\prime}, \ldots, x_{m}^{\prime}\right) \prod_{i=1}^{n} \overline{g\left(x_{i}, y_{i}\right)} \prod_{i=1}^{m} g\left(x_{i}^{\prime}, y_{i}^{\prime}\right)
\end{aligned}
$$

Now choose a family of real test densities $\left\{g_{\epsilon} \in C_{0}^{\infty}\left(M^{2}, \Omega_{1}^{2}\right)\right\}_{0<\epsilon \leq 1}$, such that the limit $\epsilon \rightarrow 0$ equals the Dirac $\delta$-distribution. Inserting these densities into Eqn. (8), the limit $\epsilon \rightarrow 0$ exists by the consideration above and yields

$$
\begin{aligned}
& \sum_{m, n} \int\left(\left(\omega_{1}\right)_{n+m}\left(\omega_{2}\right)_{n+m}\right)\left(x_{n}, \ldots, x_{1}, x_{1}^{\prime}, \ldots, x_{m}^{\prime}\right) \\
& \times \overline{f_{n}\left(x_{1}, \ldots, x_{n}\right)} f_{m}\left(x_{1}^{\prime}, \ldots, x_{m}^{\prime}\right) .
\end{aligned}
$$

Using the sequential continuity due to Theorem 2.6 we conclude that (9) is greater or equal to zero, which is the desired positivity. This finishes the proof.

Let $\omega$ denotes a state on the Borchers-Uhlmann algebra $\mathcal{B}$ and let $\left(\mathcal{H}_{\omega}, \mathcal{D}_{\omega}, \phi_{\omega}, \Omega_{\omega}\right)$ be the associated GNS-tupel. The folium of $\omega$ consists of finite convex linear combinations $\tilde{\omega}$ of states induced by vectors in $\mathcal{D}_{\omega}$ :

$$
\tilde{\omega}(A)=\operatorname{Tr}\left(\rho \pi_{\omega}(A)\right) \quad \forall A \in \mathcal{B},
$$


where $\operatorname{Tr}$ denotes the trace in $\mathcal{H}_{\omega}, \pi_{\omega}$ is the representation of $\mathcal{B}$ associated to $\omega$ and

$$
\rho=\sum_{i=1}^{N}\left|\Psi_{i}><\Psi_{i}\right| \quad \Psi_{i} \in \mathcal{D}_{\omega}
$$

is some density matrix. Contrary to the usual spectrum condition, the $\mu \mathrm{SC}$ does not characterize a distinguished state. Instead it is a property of a whole folium:

Theorem 4.5. Let $\omega$ be a state satisfying the $\mu S C$. Then the $\mu S C$ is satisfied for all states in the folium of $\omega$.

Proof. Consider a state $\tilde{\omega}$ in the folium of $\omega$. All m-point distributions $\tilde{\omega}_{m}$ of $\tilde{\omega}$ are finite linear combinations of $l+m$-point distributions of $\omega$ smeared with suitable test functions from both ends, i.e.,

$$
\tilde{\omega}_{m}\left(x_{1}, \cdots, x_{m}\right)=\sum_{l} \omega_{l+m}\left(f_{j_{1}}, \cdots, f_{j_{k}}, x_{1}, \cdots, x_{m}, f_{j_{k+1}}, \cdots, f_{j_{l}}\right) .
$$

It is therefore sufficient to show that for all $m \in \mathbb{N}$

$$
\Gamma:=\mathrm{WF}\left(\omega_{l+m}\left(f_{j_{1}}, \cdots, f_{j_{k}}, x_{1}, \cdots, x_{m}, f_{j_{k+1}}, \cdots, f_{j_{l}}\right)\right) \subseteq \Gamma_{m},
$$

where $\Gamma_{m}$ was defined in Definition 4.1 above.

Using Corollary 2.8 we obtain for the l.h.s. of (11)

$$
\begin{aligned}
& \Gamma \subseteq\left\{\left(x_{1}, k_{1} ; \cdots ; x_{m}, k_{m}\right) \in T^{*} M^{m} \backslash\{0\} \mid\right. \\
& \left.\left.\quad\left(y_{1}, 0 ; \cdots ; y_{k}, 0 ; x_{1}, k_{1} ; \cdots ; x_{m}, k_{m} ; y_{k+1}, 0 ; \cdots ; y_{l}, 0\right) \in \mathrm{WF}\left(\omega_{l+m}\right) \subset \Gamma_{l+m}\right\}_{12}\right)
\end{aligned}
$$

Moreover, since $\omega_{l+m}$ satisfies the $\mu \mathrm{SC}$ by assumption, we have for every $\left(y_{1}, 0\right.$; $\left.\cdots ; x_{i}, k_{i} ; \cdots ; y_{l}, 0\right) \in \mathrm{WF}\left(\omega_{l+m}\right)$ a corresponding graph $G_{l+m} \in \mathcal{G}_{l+m}$ together with an immersion $(x, \gamma, k)$, such that the covector fields $k_{e}$ are zero whenever $\gamma(e)$ does not connect two points in $\left\{x_{1}, \cdots, x_{m}\right\}$. For the last statement note first that the direction associated to $y_{1}$ vanishes by (12). Moreover, all causal covector fields associated to curves $\gamma$ starting at $y_{1}$ are directed towards the future by the definition of an immersion, hence using property (ii) of (7), $k_{e}=0$ whenever $\gamma$ starts or ends at $y_{1}$.

Consider now the point $y_{2}$. The direction associated to this point is again zero by assumption. Using the properties of the immersion and the previous result for covector fields along curves ending at $y_{1}$, one finds that the covector fields $k_{e}$ for all curves starting at $y_{2}$ are either future directed or zero. As in the previous case this implies $k_{e}=0$ for all curves starting or ending at $y_{2}$. By induction, this result extends up to $y_{k}$ and analogously from $y_{l}$ down to $y_{k+1}$. We may therefore remove from the graph $G_{l+m}$ all points $y_{1}, \cdots, y_{k}, y_{k+1}, \cdots, y_{l}$ together with all lines starting or ending at these points. The result is a graph $G_{m} \in \mathcal{G}_{m}$ together with an immersion $(x, \gamma, k)$ such that (i) and (ii) of (7) are satisfied. This completes the proof.

The following Theorem shows that our new spectrum condition is compatible with the usual Minkowski space spectrum condition.

Theorem 4.6. Let $\omega$ be a state for a quantum field theoretical model on Minkowski space, whose m-point distributions $\omega_{m}$ satisfy the Wightman axioms. Then $\omega$ satisfies the $\mu S C$. 
Proof. By assumption all m-point distributions $\omega_{m}$ satisfy the usual spectrum condition. Hence for every $m \in \mathbb{N}$ the support of the Fourier-transform of $\omega_{m}$ is contained in the following set:

$\operatorname{supp}\left(\hat{\omega}_{m}\right) \subseteq\left\{\left(k_{1}, \cdots, k_{m}\right) \in \mathbb{R}^{4 m} \mid k_{1}, k_{1}+k_{2}, \cdots, \sum_{i=1}^{m-1} k_{i} \in \bar{V}_{+}, \sum_{i} k_{i}=0\right\}=: \Gamma_{0}$.

For the corresponding wave front set we obtain by Proposition 8.1.7 of [Hör83]:

$$
\mathrm{WF}\left(\omega_{m}\right) \subseteq \mathbb{R}^{4 m} \times \Gamma_{0} \backslash\{0\} \equiv \mathbb{R}^{4 m} \times \Gamma .
$$

It is therefore sufficient to show that $\mathbb{R}^{4 m} \times \Gamma$ is contained in the r.h.s. of (7).

Let $\left(x_{1}, k_{1} ; \cdots ; x_{m}, k_{m}\right)$ be an arbitrary element in $\mathbb{R}^{4 m} \times \Gamma$. Let $G \in \mathcal{G}_{m}$ be a graph such that every vertex $i$ is connected with its next-neighbour only, i.e., $G$ is a simple chain. An immersion $(x, \gamma, k)$ of this graph into Minkowski space is given by:

1. Assign to every vertex $i$ the point $x(i):=x_{i}$.

2. The curves $\gamma_{i, j}$ are straight lines connecting $x_{i}$ and $x_{j}(j=(i-1),(i+1))$.

3. The vector fields $k_{i, i+1}$ are chosen such that $k_{i, i+1}=\sum_{j=1}^{i} k_{j}$ for all $1 \leq i \leq$ $m-1$.

Note that all vectors $k_{i, i+1}$ are in the closed forward light-cone by assumption and $k_{i} \equiv k_{i-1, i}-k_{i, i+1}$. Obviously this choice is compatible with the $\mu \mathrm{SC}$, which shows that $\left(x_{1}, k_{1} ; \cdots ; x_{m}, k_{m}\right)$ is contained in the r.h.s. of (7).

It is worth noting, that Theorem 4.6 seems to be wrong if we replace the $\mu \mathrm{SC}$ by one of its stronger versions as suggested in the footnote on page 9: Consider a linear functional satisfying the usual spectrum condition together with the property that some point $\left(x_{1}, k_{1} ; \cdots ; x_{5}, k_{5}\right) \in T^{*} M^{5} \backslash\{0\}$ with

1. $\left(x_{1}, k_{1}\right) \sim\left(x_{2}, k_{2}\right)$ and $k_{1} \in \bar{V}_{+}$,

2. $\left(x_{3}, k_{3}\right) \sim\left(x_{4}, k_{4}\right)$ and $k_{3} \in \bar{V}_{-}$and $k_{1}+k_{3} \in \bar{V}_{+}$

3. $k_{1}+k_{2}=-k_{5}$,

is in the wavefront set of its corresponding 5 -point distribution. (Compare Figure 2). For such a 5-point distribution there exists no graph $G \in \mathcal{G}_{5}$ together with an

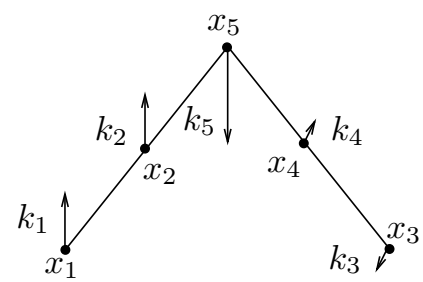

Figure 2. A counterexample

immersion, such that the edges are assigned to light-like or causal curves and such that the conditions in Definition 4.1 are satisfied with this immersion. The last statement remains true even if we use the physical requirement that this functional satisfies locality. It is not obvious whether positive linear functionals with this properties, i.e., states, exist. The latter would provide a counterexample to the stronger version of Theorem 4.6 mentioned above. On the other hand all states 
for free field models -including the Wick powers defined below-, which arise from Hadamard states, satisfy the stronger versions of our $\mu \mathrm{SC}$. One might therefore conjecture, that the fulfillment of the latter is characteristic for free field models.

\section{Definition OF Wick POLYNOMials FOR FREE FIELDS ON A MANIFOlD}

Let $\omega$ be a quasifree state of the Klein-Gordon field over a globally hyperbolic spacetime $\left(M, g_{a b}\right)$ satisfying the $\mu \mathrm{SC}$. We define a normal ordering prescription with respect to $\omega$ as on Minkowski space:

Definition 5.1. Let $\left(\mathcal{H}_{\omega}, \mathcal{D}_{\omega}, \phi, \Omega_{\omega}\right)$ be the GNS-tupel associated to $\omega$. A normal ordering prescription : : for the operator valued distributions $\phi$ is defined by the following recursion relation:

$$
\begin{aligned}
: \phi:= & \phi \\
: \phi\left(x_{1}\right) \cdots \phi\left(x_{n+1}\right):= & : \phi\left(x_{1}\right) \cdots \phi\left(x_{n}\right): \phi\left(x_{n+1}\right) \\
& -\sum_{l}: \phi\left(x_{1}\right) \cdots \hat{\phi}\left(x_{l}\right) \cdots \phi\left(x_{n}\right): \omega_{2}\left(x_{l}, x_{n+1}\right),
\end{aligned}
$$

where ${ }^{\wedge}$ means omitting the corresponding element and $\omega_{2}$ denotes the two-point distribution of the state $\omega$.

The normal product

$$
: \phi\left(x_{1}\right) \cdots \phi\left(x_{n}\right): \equiv: \phi^{\otimes n}:\left(x_{1}, \cdots, x_{n}\right)
$$

is a well defined operator valued distribution on $\mathcal{H}_{\omega}$, since the fields are smeared individually. It is symmetric in its arguments. For the subsequent definition of Wick powers, i.e., for the restriction of Eqn. (13) to the diagonal, some auxiliary operators are needed:

5.1. Auxiliary Wick monomials. Let $\Delta \in \mathcal{D}^{\prime}\left(M^{n} \times M\right)$ be a distribution, such that for all smooth test densities $f=f\left(x_{1}, \cdots, x_{n} ; x\right) d \mu_{1} \cdots d \mu_{n} d \mu \in C_{0}^{\infty}\left(M^{n} \times\right.$ $\left.M, \Omega^{n+1}\right)$

$$
\Delta(f) \equiv \int f(x, \cdots, x ; x) d \mu
$$

It is obvious that $\Delta$ is properly supported. For its wave front set one finds -using the fact that on Minkowski space $\Delta$ is a product of Dirac $\delta$ distributions-

$$
\begin{gathered}
\mathrm{WF}(\Delta)=\left\{\left(x, k_{1} ; \cdots ; x, k_{n} ; x, k\right) \in T^{*} M^{n+1} \backslash\{0\} \mid \sum k_{i}=-k\right\} \\
\mathrm{WF}_{M^{n}}(\Delta)=\left\{\left(x, k_{1} ; \cdots ; x, k_{n}\right) \in T^{*} M^{n} \backslash\{0\} \mid \sum k_{i}=0\right\}
\end{gathered}
$$

For the rest of this paper $\left\{\Delta_{\epsilon}\right\}_{0<\epsilon<1}$ will denote a family of smooth operators from $C_{0}^{\infty}\left(M, \Omega_{1}\right)$ to $\mathcal{D}^{\prime}\left(M^{n}\right)$ converging to $\Delta$ in $\mathcal{D}^{\prime}\left(M^{n} \times M\right)$ if $\epsilon \rightarrow 0$. Without loss of generality we will further assume that all operators $\Delta_{\epsilon}$ are real.

Definition 5.2. Given the normal ordering prescription and the family $\left\{\Delta_{\epsilon}\right\}$ of smooth operators we define the auxiliary Wick monomials to be the following operator valued distribution

$$
: \phi_{\epsilon}^{n}:(f):=\left(: \phi^{\otimes n}: \circ \Delta_{\epsilon}\right)(f) \quad \forall f \in C_{0}^{\infty}\left(M, \Omega_{1}\right),
$$

where $\circ$ denotes composition of operators in the sense of Theorem 2.7. 
Then the Wick monomial : $\phi^{n}$ : should be an operator valued distribution on $\mathcal{H}_{\omega}$ formally defined as the limit:

$$
: \phi^{n}:(f)=\lim _{\epsilon \rightarrow 0}: \phi_{\epsilon}^{n}:(f)=\lim _{\epsilon \rightarrow 0}\left(: \phi^{\otimes n}: \circ \Delta_{\epsilon}\right)(f) \quad \forall f \in C_{0}^{\infty}\left(M, \Omega_{1}\right),
$$

It is one of the main results of this paper (See Theorem 5.7 below), to show that Eqn. (15) above can be defined rigorously and yields new Wightman fields on the manifold. To achieve this goal let us first investigate the "vacuum" expectation values for products of auxiliary Wick monomials, i.e., their m-point distributions.

Proposition 5.3. For the m-point distributions of products of auxiliary Wick monomials the limit $\epsilon \rightarrow 0$ exists in the sense of sequentially continuous convergence for distributions. For the corresponding kernels one finds:

$$
\begin{aligned}
& \mathcal{W}_{m}^{\left(n_{1}, \cdots, n_{m}\right)}\left(x_{1}, \cdots, x_{m}\right) \\
& :=\lim _{\epsilon_{1} \cdots \epsilon_{m} \rightarrow 0}\left(\Omega,: \phi_{\epsilon_{1}}^{n_{1}}:\left(x_{1}\right) \cdots: \phi_{\epsilon_{m}}^{n_{m}}:\left(x_{m}\right) \Omega\right) \\
& =\sum_{\substack{a_{i, j} \\
1 \leq i<j \leq m}}\left[\prod_{1 \leq i<j \leq m} \frac{\omega_{2}\left(x_{i}, x_{j}\right)^{a_{i, j}}}{a_{i, j} !}\right] n_{1} ! n_{2} ! \cdots n_{m} !
\end{aligned}
$$

where $a_{i, j}$ is the matrix containing the number of pairings between points $x_{i}$ with $x_{j}(i, j=1, \ldots, m)$ and the sum is taken over all possible choices of the $a_{i, j}$ such that $\sum_{i} a_{i, j}=n_{j}$ for all $j=1, \ldots, m$.

Proof. We refrain from giving full details of the proof since the combinatorial arguments are well-known (see, e.g., Hep69]). The only crucial point is to show that the r.h.s of Eqn. (16) which contains products of two-point distributions is well defined, since -by Theorem 2.6- we have sequential continuity whenever the limit exists. On the other hand the existence of the limit is an immediate consequence of Theorem 4.4, which applies, since all quasifree Hadamard states satisfy the $\mu \mathrm{SC}$.

We will show below that the m-point distributions $\mathcal{W}_{m}^{\left(n_{1}, \cdots, n_{m}\right)}$ for all $n_{i} \leq N$, $1 \leq i \leq m$ satisfy positivity and local commutativity, i.e., they are Wightman distributions. Now every hierarchy of Wightman distributions gives rise to a state on the corresponding Borchers-Uhlmann algebra satisfying local commutativity. Hence we may apply the GNS-reconstruction Theorem to obtain a GNS-tupel $\left(\mathcal{H}_{N}, \mathcal{D}_{N},: \phi^{1}:, \ldots,: \phi^{N}:, \Omega_{N}\right)$, which contains the Wick monomials $: \phi^{1}:, \ldots$, $: \phi^{N}$ : as Wightman fields on $\mathcal{H}_{N}$ with dense invariant domain $\mathcal{D}_{N}$. Since $: \phi: \equiv \phi$, one obviously has $\mathcal{H}_{\omega} \subseteq \mathcal{H}_{N}$ and $\mathcal{D}_{\omega} \subseteq \mathcal{D}_{N}$, where $\mathcal{H}_{\omega}$ and $\mathcal{D}_{\omega}$ are the GNS-Hilbert space and dense invariant domain for the original field $\phi$ in the state $\omega$ respectively. Now $\mathcal{D}_{\omega}$ is already dense in $\mathcal{H}_{N}$, since for every $\Psi \in \mathcal{D}_{N}$ there exists a sequence of vectors in $\mathcal{D}_{\omega}$ converging to $\Psi$. To obtain the latter replace all Wick monomials in the expression for $\Psi$ by their corresponding auxiliary Wick monomials. The convergence of the sequence obtained in that way is an immediate consequence of the continuity property of the corresponding m-point distribution stated in Proposition 5.3 above. We conclude that $\mathcal{H}_{N}$ and $\mathcal{H}_{\omega}$ and $\Omega_{\omega}$ and $\Omega_{N}$ respectively are equal, which means that the Wick monomials can be represented as Wightman fields in the Hilbert space $\mathcal{H}_{\omega}$. The subspace $\mathcal{D}_{\omega}$ may not be an invariant domain for the Wick monomials in general, but due to the following Theorem, it is always a 
core for the closure of the Wick monomials with respect to the "universal" domain $\mathcal{D} \equiv \cup \mathcal{D}_{N}$. This result ensures that we "do not loose information" by restricting the Wick monomials to the subspace $\mathcal{D}_{\omega}$.

Theorem 5.4. The domain $\mathcal{D}_{\omega}$ is a core for the closure of any Wick monomial $: \phi^{n}:$ with respect to $\mathcal{D}$.

Proof. To prove the Theorem, we have to show that for all test densities $f \in$ $C_{0}^{\infty}\left(M, \Omega_{1}\right)$ the closure of : $\phi^{n}:(f)$ restricted to $\mathcal{D}_{\omega}$ coincides with the closure of : $\phi^{n}$ : $(f)$ defined on $\mathcal{D}$. We note first that all Wick monomials are hermitian, hence their closure with respect to $\mathcal{D}$ exist. Consider an arbitrary element $\left\{: \phi^{n}:(f) \Psi, \Psi\right\} \in$ $\overline{\Gamma\left(: \phi^{n}:(f)\right)}$ in the closure of the graph of $: \phi^{n}:(f)$, where $f$ is a test density as above. We will show below that there exists a sequence $\tilde{\Psi}_{j} \in \mathcal{D}_{\omega}$ converging to $\Psi \in \mathcal{D}$, such that $: \phi^{n}:(f) \tilde{\Psi}_{j}$ converges to $: \phi^{n}:(f) \Psi$.

Let $\left\{\Psi_{j}\right\}_{j \in \mathbb{N}}$ be a sequence of vectors in $\mathcal{D}$ converging to $\Psi$, such that : $\phi^{n}:(f) \Psi_{j}$ converges to : $\phi^{n}:(f) \Psi$. Our assumption guarantees the existence of such a sequence and that every $\Psi_{j}$ is a polynomial of Wick monomials applied to the GNS-vacuum. Replacing every Wick monomial $: \phi^{m}$ : in the expression for $\Psi_{j}$ by its corresponding auxiliary Wick monomial $: \phi_{\delta}^{m}$ : yields elements $\Psi_{j, \delta} \in \mathcal{D}_{\omega}$ which converge to $\Psi_{j}$ for $\delta \rightarrow 0$. Now let $\epsilon>0$ be given. By assumption there exists some $j$ with

$$
\left\|\Psi_{j}-\Psi\right\|<\frac{\epsilon}{2}, \quad\left\|: \phi^{n}:(f)\left(\Psi_{j}-\Psi\right)\right\|<\frac{\epsilon}{2} .
$$

Moreover, due to the continuity property of the m-point distribution for the auxiliary Wick monomials (Proposition 5.3 above), there exists a $\delta>0$, such that

$$
\left\|\Psi_{j, \delta}-\Psi_{j}\right\|<\frac{\epsilon}{2}, \quad\left\|: \phi^{n}:(f)\left(\Psi_{j, \delta}-\Psi_{j}\right)\right\|<\frac{\epsilon}{2}
$$

Combining the two previous results we get

$$
\left\|\Psi_{j, \delta}-\Psi\right\| \leq \epsilon \quad\left\|: \phi^{n}:(f)\left(\Psi_{j, \delta}-\Psi\right)\right\| \leq \epsilon
$$

hence, $\left\{: \phi^{n}:(f) \Psi, \Psi\right\} \in \overline{\Gamma\left(: \phi^{n}:(f)\left\lceil\mathcal{D}_{\omega}\right)\right.}$, which proves the assertion.

Let us now finish the argument by showing that the linear functionals $\mathcal{W}_{m}^{\left(n_{1}, \cdots, n_{m}\right)}$ are Wightman distributions.

Lemma 5.5 (Positivity). The hierarchy of m-point distributions for the Wick monomials satisfy positivity, i.e.,

$$
\begin{aligned}
\sum \int & \cdots \int \bar{f}_{j}\left(x_{1}, \ldots, x_{j}\right) \\
& \times \mathcal{W}_{j+k}^{\left(m_{j}, \ldots, m_{1}, n_{1}, \ldots, n_{k}\right)}\left(x_{j}, \ldots, x_{1}, y_{1}, \ldots, y_{k}\right) f_{k}\left(y_{1}, \ldots, y_{k}\right) \geq 0,
\end{aligned}
$$

for all finite sequences $f_{0}, f_{1}\left(x_{1}\right), f_{2}\left(x_{1}, x_{2}\right), \ldots$ of test densities. As usual the upper bar denotes complex conjugation.

Proof. The fields : $\phi^{\otimes n}$ : obviously are (hermitian) Wightman fields. Let us denote their Wightman M-point distributions by

$$
\begin{aligned}
& \mathcal{W}_{M=\sum n_{i}}^{\left(\otimes n_{1}, \cdots, \otimes n_{m}\right)}\left(x_{1_{1}}, \ldots, x_{1_{n_{1}}}, \ldots, x_{m_{1}}, \ldots, x_{m_{n_{m}}}\right) \\
&:=\left(\Omega,: \phi^{\otimes n_{1}}:\left(x_{1_{1}}, \ldots, x_{1_{n_{1}}}\right) \cdots: \phi^{\otimes n_{m}}:\left(x_{m_{1}}, \ldots, x_{m_{n_{m}}}\right) \Omega\right) .
\end{aligned}
$$

The corresponding hierarchy satisfies positivity and local commutativity by definition. 
Consider now a finite sequence of test densities $\left\{f_{i}\left(x_{1}, \ldots, x_{i}\right)\right\}$. We may use the operators $\Delta_{\epsilon}$ introduced above to map every $f_{i}$ into an admissible test density $\Delta_{\epsilon} \circ f_{i}$ for : $\phi^{\otimes n}:$. It follows that

$$
\begin{aligned}
& 0 \leq \sum \int \cdots \int \overline{m_{1} \Delta_{\epsilon} \cdots^{m_{j}} \Delta_{\epsilon} \circ f_{j}}\left(x_{1_{1}}, \ldots, x_{1_{m_{1}}}, \ldots, x_{j_{1}}, \ldots, x_{j_{m_{j}}}\right) \\
& \times \mathcal{W}_{J+K}^{\left(\otimes m_{j}, \cdots, \otimes m_{1}, \otimes n_{1}, \cdots, \otimes n_{k}\right)}\left(x_{j_{n_{j}}}, \ldots, x_{1_{1}}, y_{1_{1}}, \ldots, y_{k_{n_{k}}}\right) \\
& \times{ }^{n_{1}} \Delta_{\epsilon} \ldots{ }^{n_{k}} \Delta_{\epsilon} \circ f_{k}\left(y_{1_{1}}, \ldots, y_{1_{n_{1}}}, \ldots, y_{k_{1}}, \ldots, y_{k_{n_{k}}}\right)
\end{aligned}
$$

where the upper left index on ${ }^{n} \Delta_{\epsilon}$ indicates the image space $\mathcal{D}^{\prime}\left(M^{n}\right)$ of the corresponding operator and $J$ and $K$ abbreviate $\sum m_{i}$ and $\sum n_{i}$ respectively. We now insert Eqn. (18) together with Eqn. (14) and use the symmetry of the Wick ordering prescription as well as the fact that all operators $\Delta_{\epsilon}$ are real. We obtain:

$$
\begin{aligned}
(19)=\sum & \int \cdots \int \bar{f}_{j}\left(x_{1}, \ldots, x_{j}\right) \\
& \times\left(\Omega,: \phi_{\epsilon}^{m_{j}}:\left(x_{j}\right) \cdots: \phi_{\epsilon}^{m_{1}}:\left(x_{1}\right): \phi_{\epsilon}^{n_{1}}:\left(y_{1}\right) \cdots: \phi_{\epsilon}^{n_{k}}:\left(y_{k}\right) \Omega\right) \\
& \times f_{k}\left(y_{1}, \ldots, y_{k}\right)
\end{aligned}
$$

Note that by Proposition 5.3 the expression above converges to the l.h.s. of Eqn. (17) in the limit $\epsilon \rightarrow 0$, which finishes the proof.

The locality for the m-point distributions $\mathcal{W}_{m}^{\left(n_{1}, \cdots, n_{m}\right)}$ is the result of the following

Lemma 5.6 (Locality). The m-point distributions $\mathcal{W}_{m}^{\left(n_{1}, \cdots, n_{m}\right)}$ obey locality, i.e., for $f_{1}, \ldots, f_{m} \in C_{0}^{\infty}\left(M, \Omega_{1}\right)$, such that the supports of $f_{i}$ and $f_{i+1}$ are space-like separated,

$$
\mathcal{W}_{m}^{\left(n_{1}, \cdots, n_{m}\right)}\left(f_{1} \otimes \cdots f_{i} \otimes f_{i+1} \otimes \cdots f_{m}\right)=\mathcal{W}_{m}^{\left(n_{1}, \cdots, n_{m}\right)}\left(f_{1} \otimes \cdots f_{i+1} \otimes f_{i} \otimes \cdots f_{m}\right)
$$

Proof. As in the proof of the previous Theorem, we prove the statement for the auxiliary Wick monomials first:

Let $f_{i}$ and $f_{i+1}$ be two test densities with space-like separated supports. Applying the appropriate operator $\Delta$ to $f_{i}$ and $f_{i+1}$ yields test densities ${ }^{n_{i}} \Delta\left(f_{i}\right)$ and ${ }^{n_{i+1}} \Delta\left(f_{i+1}\right)$ whose supports are space-like separated, too. Hence one can find two space-like separated closed bounded regions $U_{n_{i} \Delta\left(f_{i}\right)}$ and $U_{n_{i+1} \Delta\left(f_{i+1}\right)}$ strictly containing the supports of ${ }^{n_{i}} \Delta\left(f_{i}\right)$ and ${ }^{n_{i+1}} \Delta\left(f_{i+1}\right)$ respectively. This implies the existence of a subsequence of smooth operators ${ }^{n_{i}} \Delta_{\epsilon},{ }^{n_{i+1}} \Delta_{\delta}$ converging to ${ }^{n_{i}} \Delta$ and ${ }^{n_{i+1}} \Delta$ respectively, such that $\operatorname{supp}^{n_{i}} \Delta_{\epsilon}\left(f_{i}\right)$ and $\operatorname{supp}^{n_{i+1}} \Delta_{\delta}\left(f_{i+1}\right)$ are spacelike separated for all couples $\epsilon, \delta$. Using locality for the original fields $\phi$ we conclude:

$$
\begin{aligned}
\left(\Omega,: \phi_{\rho}^{n_{1}}:\left(f_{1}\right) \cdots: \phi_{\epsilon}^{n_{i}}:\left(f_{i}\right): \phi_{\delta}^{n_{i+1}}:\left(f_{i+1}\right) \cdots: \phi_{\rho}^{n_{m}}:\left(f_{m}\right) \Omega\right) \\
\quad=\left(\Omega,: \phi_{\rho}^{n_{1}}:\left(f_{1}\right) \cdots: \phi_{\delta}^{n_{i+1}}:\left(f_{i+1}\right): \phi_{\epsilon}^{n_{i}}:\left(f_{i}\right) \cdots: \phi_{\rho}^{n_{m}}:\left(f_{m}\right) \Omega\right) .
\end{aligned}
$$

By the continuity result of Proposition 5.3 we may pass to the limit $\rho, \epsilon, \delta \rightarrow 0$, obtaining

$$
\mathcal{W}_{m}^{\left(n_{1}, \cdots, n_{m}\right)}\left(f_{1} \otimes \cdots f_{i} \otimes f_{i+1} \otimes \cdots f_{m}\right)=\mathcal{W}_{m}^{\left(n_{1}, \cdots, n_{m}\right)}\left(f_{1} \otimes \cdots f_{i+1} \otimes f_{i} \otimes \cdots f_{m}\right) .
$$

Combining all the results obtained and using the GNS-reconstruction Theorem we have thus proved the following 
Theorem 5.7. The Wick monomials of the free Klein-Gordon field on a globally hyperbolic spacetime with respect to any quasifree state $\omega$ satisfying the $\mu S C$ are well defined Wightman fields on the GNS-Hilbert space of $\omega$ with core $\mathcal{D}_{\omega}$ and dense invariant domain $\mathcal{D}$ generated by applying finitely many smeared Wick monomials to $\Omega$.

Remark .

- Our construction of Wick monomials generalizes by linearity to polynomials which may also contain derivatives of the field. This is due to the fact that derivations do not enlarge the wave front set of a distribution (see the Remark in section 2).

- It should be possible to generalize the results of this section to other free field examples, e.g., the free Dirac- or electro-magnetic field.

It is worth noting that the GNS-vacuum $\Omega$, which was used in the normal ordering prescription above, simultaneously yields an admissable state for the Wick monomials in the sense of

Theorem 5.8. Every state $\omega^{(n)}$ given by the Wightman distributions of the Wick monomials : $\phi^{n}:($ any $n \in \mathbb{N})$ satisfies the $\mu S C$.

Proof. The m-point distributions $\omega_{m}^{(n)}$ are "vacuum" expectation values of product of Wick monomials in the sense of Proposition 5.3 by construction:

$$
\omega_{m}^{(n)}\left(x_{1}, \cdots, x_{m}\right)=\sum_{\substack{a_{i, j} \\ 1 \leq i<j \leq m}}\left[\prod_{1 \leq i<j \leq m} \frac{\omega_{2}\left(x_{i}, x_{j}\right)^{a_{i, j}}}{a_{i, j} !}\right](n !)^{m}
$$

which implies that $\operatorname{WF}\left(\omega_{m}^{(n)}\right)$ is contained in the following set:

$$
\mathrm{WF}\left(\omega_{m}^{(n)}\right) \subseteq \bigcup_{p \subset P} \bigoplus_{r \in p}\left[\mathrm{WF}\left(\omega_{2}^{r}\right) \oplus \mathrm{WF}\left(\omega_{2}^{r}\right)\right]
$$

where $p$ runs over the nonempty subsets of the set of all ordered pairs $P=$ $\{(i, j), 1 \leq i<j \leq m\}$. Note that we are using the same notation as in Eqn. (6) and that the sum of two wave front sets of the two-point distribution over the same base points is stable under further sums. Moreover the two-point distribution $\omega_{2}$ satisfies the $\mu \mathrm{SC}$ by assumption and the latter is conic by definition. Hence we can apply verbatim the argumentation in the proof of Proposition 4.3 which completes the proof of the Theorem.

We end this paper by stating the following straightforward result on the energymomentum tensor for free fields on a manifold.

Corollary 5.9. The normal ordered energy-momentum tensor $T_{\mu \nu}$ of a free massive scalar Klein-Gordon field on a globally hyperbolic manifold is a Wightman field on the GNS-Hilbert space for any quasifree state $\omega$ satisfying the $\mu S C$.

Proof. The normal ordered energy-momentum tensor is by definition a (real) Wick polynomial of the corresponding free field. Hence the assertion follows immediately from Theorem 5.7 above. 


\section{Summary AND OUTLOOK}

In this paper the program sketched in [Köh95 is continued. In particular in its first part a microlocal spectrum condition $(\mu \mathrm{SC})$ for all m-point distributions of a quantum field on a curved spacetime is proposed. This condition might generalize the usual Minkowski space spectrum condition to manifolds. It is shown, that the new $\mu \mathrm{SC}$ is compatible to the former and nontrivial examples for physical states satisfying this new condition are presented. In the second part of this paper arbitrary Wick monomials for free scalar fields on globally hyperbolic spacetimes with respect to globally Hadamard states are defined rigorously. For the proofs both the $\mu \mathrm{SC}$ and the powerful methods of Hörmander's microlocal analysis are needed. In a next step they should be used to formulate causal perturbation theory on manifolds. Work is in progress in this direction and the authors do not expect any fundamental difficulties.

On Minkowski spacetime the study of wave front sets for more realistic models (e.g., QED) might result in a deeper understanding of the Wightman axioms. The free field examples considered in this paper satisfy the stronger version of our $\mu \mathrm{SC}$ suggested in the footnote on page 9 . We conjecture that this property distinguishes free field theories from interacting ones. We are confident that this conjecture can be proved at the level of perturbation theory.

Acknowledgement . The authors have benefited from discussions with many members of the II. Institut für Theoretische Physik at the University of Hamburg. R.B. gratefully acknowledges financial support of the Graduiertenkolleg of the University of Hamburg during his stay in Germany. M.K. is supported by the DFG and thanks the INFN for financial support and hospitality during his stay in Naples. Special thanks are due to M. Radzikowski for interesting discussions during his visit in Hamburg.

\section{REFERENCES}

[Bor62] H. J. Borchers. On the structure of the algebra of the field operators. Nuovo Cimento, 24:214, 1962.

[DB60] B. S. DeWitt and R. W. Brehme. Radiation damping in a gravitational field. Ann. Phys., 9:220-259, 1960.

[DH72] J. J. Duistermaat and L. Hörmander. Fourier integral operators II. Acta Math., 128:183, 1972.

[Dim80] J. Dimock. Algebras of local observables on a manifold. Comm. Math. Phys., 77:219$228,1980$.

[Dim82] J. Dimock. Dirac quantum fields on a manifold. Trans. Am. Math. Soc., 269:133-147, 1982.

[Dim92] J. Dimock. Quantized electromagnetic field on a manifold. Rev. Math. Phys., 4:223-233, 1992.

[Dui73] J. J. Duistermaat. Fourier Integral Operators. Courant Institute of Mathematical Sciences, New York University, 1973.

[FH87] K. Fredenhagen and R. Haag. Generally covariant quantum field theory and scaling limit. Comm. Math. Phys., 108:91, 1987.

[Fre92] K. Fredenhagen. On the general theory of quantized fields. In K. Schmüdgen, editor, Mathematical Physics X, pages 136-152, Berlin, 1992. Springer Verlag.

[FSW78] S. A. Fulling, M. Sweeny, and R. M. Wald. Singularity structure of the two-point function in quantum field theory in curved spacetime. Com. Math. Phys., 63:257-264, 1978.

[Ful89] S. A. Fulling. Aspects of Quantum Field Theory in Curved Space-Time. Cambridge University Press, Cambridge, 1989. 
[Haa92] R. Haag. Local quantum physics: Fields, particles, algebras. Springer, Berlin, Germany, 1992.

[Hep69] K. Hepp. Théorie de la renormalisation. Number 2 in Lecture Notes in Physics. Springer Verlag, Berlin,Heidelberg, 1969.

[HK64] R. Haag and D. Kastler. An algebraic approach to quantum field theory. J. Math. Phys., 5:848, 1964.

[HNS84] R. Haag, H. Narnhofer, and U. Stein. On quantum field theory in gravitational background. Comm. Math. Phys., 94:219-238, 1984.

[Hör71] L. Hörmander. Fourier integral operators I. Acta Math., 127:79, 1971.

[Hör83] L. Hörmander. The Analysis of Linear Partial Differential Operators I. Springer, Berlin, 1983.

[Jun95] W. Junker. Adiabatic vacua and Hadamard states for scalar quantum fields on curved spacetimes. PhD thesis, University of Hamburg, 1995.

[Köh95] M. Köhler. New examples for Wightman fields on a manifold. Class. Quant. Grav., 12:1413-1427, 1995

[KW91] B. S. Kay and R. M. Wald. Theorems on the uniqueness and thermal properties of stationary, nonsingular, quasifree states on spacetimes with a bifurcate Killing horizon. Phys. Rep., 207(2):49-136, 1991.

[Rad92] M. J. Radzikowski. The Hadamard condition and Kay's conjecture in (axiomatic) quantum field theory on curved space-time. PhD thesis, Princeton University, October 1992.

[Sat69] M. Sato. Hyperfunctions and partial differential equations. In Proc. Int. Conf. on Funct. Anal. and Rel. Topics, pages 91-94, Tokyo, 1969. Tokyo University Press.

[Sat70] M. Sato. Regularity of hyperfunction solution of partial differential equations. Actes Congr. Int. Math. Nice, 2:785-794, 1970.

[Uhl62] A. Uhlmann. Über die Definition der Quantenfelder nach Wightman und Haag. Wiss. Zeitschrift Karl Marx Univ., 11:213, 1962.

[Ver94] R. Verch. Local definiteness, primarity and quasiequivalence of quasifree Hadamard quantum states in curved spacetime. Comm. Math. Phys, 160:507-536, 1994.

[Wal78] R. M. Wald. Trace anomaly of a conformally invariant quantum field in curved spacetime. Phys. Rev. D, 17(6):1477-1484, 1978

[Wal94] R. M. Wald. Quantum field theory in curved spacetime and black hole thermodynamics. Chicago lectures in physics. Univ. Chicago Press, Chicago, USA, 1994.

[WG64] A. S. Wightman and L. Gårding. Fields as operator valued distributions in relativistic quantum theory. Ark. Fys, 23(13), 1964.

[Wol92] M. Wollenberg. Scaling limits and type of local algebras over curved spacetime. In W. B. Arveson et al., editors, Operator algebras and topology. Putman Research notes in Mathematics 270, Harlow: Longman, 1992. 